\title{
Ricerche del 2008-2010 nella villa maritima di San Simone/Simonov zaliv presso Isola/Izola (Slovenia)
}

\author{
Stefan Groh and Helga Sedlmayer
}

In the villa maritima of Simonov zaliv near Izola (Slovenia) new archaeological research were carried out in the years 2008-20I0. Apart from new mappings of visible building remains, the installation of a GIS-project and geophysical measurements, excavations were conducted in two areas of the villa. In 2008 the archaeological research was focused on the building complex in the north, in 2009-2010 a part of the porticus near the port was excavated. The results of the stratigraphical excavations and analyses of finds give important new information about the chronology and building concept of the villa during the $\mathrm{I}^{\mathrm{st}}$ century BC and the $\mathrm{I}^{\mathrm{st}}$ century AD.

Keywords: villa maritima, San Simone/Simonov zaliv, excavations, analysis of finds, chronology

N

ella villa maritima di San Simone/Simonov zaliv, presso Isola/Izola sulla costa adriatica della Slovenia, sono iniziate delle ricerche sistematiche, grazie ad una cooperazione di ricerca tra l'Inštitut za dediščino Sredozemlja der Znanstveno-raziskovalno središče Koper Univerze na Primorskem (UP ZRS) e l>Österreichisches Archäologisches Institut Wien (ÖAI). Scopo di questo accordo di cooperazione è di acquisire nuove conoscenze circa la topografia, le strutture edilizie e la cronologia relative alla villa. Di seguito si presentano i risultati delle prime campagne di ricerca 200820I0, ottenuti con $1>$ installazione di un sistema geoinformativo e di misurazioni geodetiche dei resti scavati e restaurati nel XX secolo, con misurazioni geofisiche e con scavi stratigrafici mirati.
Indagini archeologiche sistematiche nella villa ebbero luogo negli anni Venti del XX secolo, sotto la direzione di Attilio Degrassi e di Bruna Tamaro e negli anni Cinquanta sotto quella di Vinko Šribar. Nella seconda meta del XX secolo furono effettuati mappature dei moli subacquei nel bacino portuale, le prime misurazioni geofisiche e scavi archeologici da Giordano Labud sotto la direzione di Marko Stokin. ${ }^{2} \mathrm{Nel}$

Attilio Degrassi, »Tracce di Roma sulla spiaggia di San Simone, « Archeografo Triestino Io, no. 3 (1923).

2 Michael Donderer, Die Chronologie der römischen Mosaiken in Venetien und Istrien bis zur Zeit der Antonine (Berlin: Mann, 1986); Elica Boltin-Tome, $\gg$ Arheološke najdbe na kopnem in na morskem dnu v Viližan in Simonovem zalivu v Izoli,« Annales I (1991); Federica Fontana, La villa romana di Barcola: A proposito delle Villae Maritimae della Regio X (Roma: Quasar, 1993); Giordano Labud, »Studio tipilogico di reperti anforici rinvenuti nel sito archeologico romano di San Simone (Simonov zaliv), nell'Istria settentrionale, « Opuscula romana 20 (1996); Iva Mikl Curk, »Simonov zaliv - Terra Sigillata from the First Field Campaigns, « Opuscula romana 20 (1996); 
2006 Branko Mušič effettuò misurazioni geofisiche (particelle I419-1429).

La villa maritima di San Simone/Simonov zaliv si trova su una penisola oggi larga circa 120 $\times$ I50 $\mathrm{m}$ e possiede nella parte sudoccidentale un porto provvisto di due moli della grandezza di circa $7400 \mathrm{~m}^{2} .{ }^{4}$ La parte nordorientale della penisola è delimitata da un ruscello, a sud della penisola, ed a est dei moli, si trovava una depressione del terreno, minacciata dalle inondazioni. Tra gli scavi del 1922 e quelli della metà del XX secolo la riva fu asportata dal mare in questo punto per una lunghezza di $15-20 \mathrm{~m} ;{ }^{5}$ in seguito a tale evento, nel $1968 \mathrm{fu}$ disposto un argine fino al molo romano meridionale. Questi terrapieni e l'originario andamento della costa si riconoscono grazie alle foto più vecchie ${ }^{6} \mathrm{e}$ in base alle prospezioni geofisiche, condotte dall'ÖAI 2008.

Scopo degli scavi del 2008-20ı0 è stato quello di acquisire nuove e sicure informazioni sulla cronologia della villa e di verificare i dati delle prospezioni geofisiche, condotte da B. Mušič e dall'ÖAI. Secondo le prospezioni geofisiche a un corpo in parte già scavato a nord segue una costruzione simile a un corridoio largo circa $6 \mathrm{~m}$ intorno a un cortile ampio almeno $27 \mathrm{~m}$. Questo poi porta a una porticus, parzialmente indagata, nella parte meridionale della villa. ${ }^{7}$

\section{Ricerche tra il complesso edilizio a nord e a sud della villa}

Gli scavi del 2008 si sono estesi su una superficie di oltre $3 \times 8 \mathrm{~m}$, ovvero $24 \mathrm{~m}^{2}$, congiungendosi sia agli scavi anteriori nel complesso edilizio a

Snježana Karinja, »Dve rimski pristanišči v Izoli,« in Arheoloska istraživanja u Istri, ed. Božidar Čečuk, (Zagreb: Hrvatsko arheološko društvo, 1997); Marina De Franceschini, Le ville romane della Regio X (Venetia et Histria) (Roma: »L'Erma « di Bretschneider, 1998); Marko Stokin, Simonov zaliv: Antična vila « Annales, Series historia et sociologia $\mathrm{I}$, no. I (2001).

3 Branko Mušič, Poročilo o geofizikalni raziskavi: Izola - Simonov zaliv (Ljubljana: Inedito rapporto di prospezione, 2006).

4 Attilio Degrassi, »I porti romani dell’Istria, « in Scritti di Archeologia e di Antichità Classiche in onore di Carlo Anti (Firenze: Sansoni, 1955).

Vinko Sribar, »Constatations archéologico-topographiques dans la baie de Simon près d'Izola, «Arheoloski Vestnik 9-10 (1958-1959).

6 Karinja, Dve rimski pristanišči v Izoli.

7 Stokin, Simonov zaliv; Poroćilo o geofizikalni raziskavi. nord della villa che a un contesto della costruzione meridionale.

I risultati dell'indagine archeologica $2008^{8}$ permettono di articolare la storia edilizia dell'area di scavo in quattro periodi, che descrivono attività con forte impatto sull'ambiente e sulle strutture edilizie.

Nel periodo i ha luogo la preparazione del cantiere e la predisposizione di spianamenti allo scopo di ottenere un livello il più possibile unitario. Dal livello di partenza e di lavoro (periodo I.I-2) comincia la costruzione delle fosse e dei muri di fondazione (periodo I.3).

Nel periodo 2 segue alla costruzione dei muri la sistemazione dei vani $(\mathrm{A}-\mathrm{C})$. Il vano $\mathrm{A}$ a nord è largo 4, I $\mathrm{m}$ (da nord a sud) e largo per lo meno $3 \mathrm{~m}$. I rinvenimenti del periodo d'uso sono documentabili solo in pochi casi a causa delle numerose fosse, cronologicamente più recenti, e di quelle relative all'orizzonte di abbandono (periodo 3). Del pavimento a mosaico bianco del vano A rimane appena una superficie di $0,06 \mathrm{~m}^{2}$. Dal punto di vista della tecnica di costruzione il vano A appartiene al complesso edilizio settentrionale della villa messo parzialmente in luce da Attilio Degrassi, cui corrisponde anche per l'arredo di qualità con pavimenti musivi.

Gli ambiti B e C risultano dall'aggiunta del

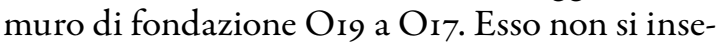
risce ad angolo retto, ma ha, come tutte le strutture scavate nel corpo meridionale della villa, un orientamento divergente di circa $5^{\circ}$ verso ovest. Nell'area B si costruisce un pozzo nero $\left(\mathrm{O}_{2} \mathrm{O}\right)$, parzialmente rimesso in luce nell'angolo sudoccidentale dello scavo.

Nel periodo 3 sono abbandonati i vani scavati. Questo abbandono procede di pari passo con il reimpiego del materiale da costruzione (blocchi dell'opera muraria, pavimenti mosaicati). La spoliazione della villa che portò al suo abbandono sembra esser stata sistematica.

8 Stephan Groh and Helga Sedlmayer, »Die Grabungen in der römischen Villa maritima von San Simone/Simonov zaliv bei Isola/ Izola, Slowenien, « Annales, Series historia et sociologia 18 no. 2 (2008); Stephan Groh and Helga Sedlmayer, »Nuove ricerche nella villa maritima romana di San Simone/Simonov zaliv presso Isola/Izola (Slovenia), «Aquileia Nostra 80 (2010). 
Il periodo 4 corrisponde al degrado postantico ovvero all'uso agricolo del terreno.

Circa 400 reperti sono considerati significativi dalla sezione di scavo dell'anno 2008. La metà di essi proviene dagli strati che stanno in rapporto immediato con le preparazioni, con I'utilizzo e con l'abbandono del complesso. L'altro 50 per cento viene dai livelli che furono alterati oppure distrutti in epoca postantica; in questo caso compaiono numerosi residui antichi accanto a un numero ridotto di rinvenimenti della prima età moderna e di epoca contemporanea.

\section{Ricerche nel complesso edilizio a sud della villa}

Per chiarire le fasi costruttive nella zona della porticus nel 2009-2010 si attuò un saggio di scavo ad oriente dell'area di scavo già indagata da M. Stokin. I livelli antichi erano stati disturbati dalle molteplici attività di scavo del recente passato e tagliati all'inizio dell'età moderna da un sentiero, che congiungeva San Simone con il porto. Nonostante ciò, i resti antichi della porticus furono rinvenuti in buon stato di conservazione. Ciò è dovuto al fatto che l'obliterazione delle costruzioni del I sec. d.C. fu realizzata attraverso sistematici lavori di riporto e livellamento.

Prima della costruzione della porticus l'area vicino al porto fu usata per lo scarico di rifiuti. Diversi strati di riporto, contenenti per lo più resti di anfore, sono stati messi in luce sotto il livello di impianto della poticus. Queste azioni permisero di livellare l'iniziale forte dislivello del terreno.

La porticus ha due fasi. Nella prima fase questa era pavimentata a mosaico, nella seconda furono eretti sul lato meridionale del muro nord dei pilastri di pietra, legata da malta. Essi furono impiantati direttamente sul pavimento in mosaico. L'analisi delle strutture del portico, messe in luce negli scavi del 2009-2010, e dei materiali rinvenuti indicano che il complesso non è stato costruito prima dell'età tiberiana. Gli interventi edilizi nell'area del portico sono dunque più recenti di quelli effettuati nell'adiacente zona nord, che è stata edificata tra il 25 ed il ıo a.C.

Da un lato l'abbandono sistematico delle costruzioni e lo smontaggio dei materiali edilizi è dimostrato dal prelievo perfino delle tessere di mosaico. Dall'altro i strati di livellamento con mattoni e pietre sopra i resti delle antiche costruzioni indicano il cambiamento di funzione dell'area nell'antichità.

\section{Interpretazione e discussione dei rinvenimenti degli scavi}

2008-2010

Il tipo architettonico della villa marittima, che comprende concettualmente portici molto sporgenti, nasce in Italia nel secondo quarto del I secolo a.C.' La penisola, su cui si trova Ia villa maritima di San Simone, fu coperta da costruzioni fino al ruscello, che scorre a nordest. Muri isolati sono stati trovati anche a sud e a sudest dei moli del porto. ${ }^{\mathrm{r}} \mathrm{Da}$ ciò si potrebbe concludere che uno o più edifici principali erano situati nella penisola, ed erano fiancheggiati da un portico fino al porto. La costruzione del complesso edilizio settentrionale, cioè di un edificio principale della villa, può essere fissata con sicurezza sulla base dello scavo 2008 al periodo intorno al 25-10 a.C. La costruzione fa seguito a consistenti interventi di livellamento.

Le strutture note mediante scavi e misurazioni geofisiche indicano nella parte settentrionale e meridionale della penisola due complessi edilizi a est del porto. ${ }^{\text {II }}$ A nord è riconoscibile una fitta edificazione e, per il settore oggetto di scavo, è possibile individuare vani di alto valore qualitativo (pavimenti musivi, pitture murali); cosi il vano A, indagato nel 2008 , rivela il resto di un pavimento musivo bianco. Il complesso edilizio settentrionale di circa $40 \times 50 \mathrm{~m}$ possiede una corte interna, di circa $15 \times 22 \mathrm{~m}$, circondata da un portico. La costruzione si estende

\footnotetext{
9 Xavier Lafon, Villa Maritima (Roma: École Française de Rome, 200I); Vlasta Begović Dvoržak and Ivančica Dvoržak Schrunk, »Roman Villas in Istria and Dalmatia, Part III: Maritime Villas, « Prilozi Instituta za arheologiju u Zagrebu 2 I (2004)

Io Vinko Šribar, »Izola,«Varstvo Spomenikov 12 (1969).

I I Mušič, Poroćilo ogeofizikalni raziskavi.
} 
fino al molo marittimo. II complesso, già scavato sotto la direzione di Attilio Degrassi, oggi visibile per una superficie di $13,8 \times 18,3 \mathrm{~m}$, si trova nell'angolo meridionale di questo fabbricato settentrionale.

Nella parte meridionale della penisola è localizzato un altro complesso edilizio. Esso si compone di una grande corte interna di almeno $17 \times 36 \mathrm{~m}$ e di un portico. Singoli vani fiancheggiano a nord, ovest e sud la corte interna. La porticus è costituisce un passaggio verso il porto con vista sul mare. Il complesso edilizio misura almeno $44 \times 53 \mathrm{~m}$, ma la terminazione verso est non è nota. Nell >ambito di questo complesso meridionale si trova la corte (area B) con il pozzo nero, indagato nel 2008 , che è da interpretare come area produttiva. L $>$ appendice obliqua e la tecnica costruttiva del muro Oıg e dei vani intorno al portico meridionale, divergente del complesso settentrionale, indicano due differenti corpi di fabbrica. Il corpo meridionale diverge nel suo orientamento di circa $5^{\circ}$ verso ovest da quello settentrionale. Entrambi sono stati costruiti in una successione cronologica relativamente vicina e potrebbero aver avuto forse anche diversa funzione fra loro. I rinvenimenti degli scavi 2008 confermano ampiamente i dati relativi alle aree riconosciute come edificate nelle misurazioni geofisiche da B. Mušič e dall>ÖAI.

Negli ultimi 2000 anni il livello del mare si è innalzato di circa $1,6 \mathrm{~m}$, ciò significa che i resti dei moli marittimi che oggi giacciono al di sotto della superficie del mare e i muri di fondazione sulla terraferma, originariamente si trovavano sicuramente ad altezza maggiore rispetto al livello del mare. ${ }^{12}$ I pavimenti dell'edificio a nord della villa si trovano oggi circa I, $4 \mathrm{~m}$ sopra il livello del mare, il che corrisponde ad una altezza di circa 3 $\mathrm{m}$ sul livello del mare in epoca romana. La porticus e i vani nella parte meridionale della villa si trovano attualmente a I,I $\mathrm{m}$ al di sopra del livello

I2 Fabrizio Antonioli, Marco Anzidei, Kurt Lambeck, Rita Auriemma, Dario Gaddi, Stefano Furlani, Paola Orrù, Emanuela Solinas, Andrej Gaspari, Snježana Karinja, Vladimir Kovačić, and Luciano Surace, »Sea-level Change during the Holocene in Sardinia and in the Northeastern Adriatic (Central Mediterranean Sea) from Archaeological and Geomorphological Data, « Quaternary Science Reviews 26 (2007) del mare, quindi a un'altezza di circa 2,7 $\mathrm{m}$ sopra di esso in epoca romana. La differenza di quota tra l'edificio principale a nord e Ia porticus a sud arriva dunque a circa $0,3 \mathrm{~m}$.

La dismissione per lo meno di parte della villa si data sulla base dei dati degli scavi 20082010 verso la metà ovvero il terzo quarto del I secolo d.C. ( $50-70$ d.C.). E verosimile che gran parte del corpo settentrionale oppure forse anche dell'intero edificio principale sia stato abbandonato in quest'epoca. Questo potrebbe essere avvenuto in conseguenza di cause endogene (terremoto, maremoto o burrasche). Labbandono procede di pari passo con il reimpiego di materiale da costruzione, che in termini di cronologia assoluta ebbe luogo immediatamente dopo l'abbandono dell'edificio. L'opera sistematica di spoglio nel complesso edilizio a nord e nella porticus porta a concludere l'esistenza di interventi edilizi nelle immediate vicinanze. Lo attesta il materiale finora pubblicato rinvenuto negli scavi più vecchi nell'ambito del portico e del porto, che indica un uso di queste aree ancora nel IIl secolo d.C. ${ }^{\text {I3 }}$

\section{Summary}

Due to the topographical situation and the geophysical and archaeological findings the villa maritima of Izola/ Isola - Simonov zaliv/San Simone, situated on a peninsula, can be divided into one or more principal buildings and a portico next to the harbour.

The construction of the northern building with superbly furnished rooms, a main building of the villa, can be dated, following the results of the excavation in 2008 , in the time around $25-10 \mathrm{BC}$. The construction is carried out after extensive levelling measures.

In the south of the peninsula there is another building with a courtyard and portico, built on the passage to the harbour with sea views.

The southern structure differs in its orientation by 5 to the west from that of the northern building. The two structures have been built in a relative chronological sequence of each other. The construction of the portico in the south near the harbour is about one generation later than the construction of buildings in the north.

I3 Mikl Curk, Simonov zaliv 
The abandonment of at least parts of the villa dates, according to the results of the excavation in 2008 and 2010 around the middle or in the third quarter of the $\mathrm{I}^{\mathrm{st}}$ century AD (50-70 AD). The abandonment could be caused by endogenous influences, i.e. earthquakes or thunderstorms.

After leaving these structures of the villa, building materials (mosaic floors, walls, roof-tiles) were torn out systematically. This re-use of building material can be dated in the time immediately after the abandonment. The systematic stone robbery indicates hypothetically that the reconstruction work took place in the vicinity to the harbour, which was still in use.

\section{Povzetek}

Zaradi topografske situacije ter geofizikalnih in arheoloških najdb je mogoče obmorsko vilo v Simonovem zalivu, ki se nahaja na polotoku, razdeliti na eno ali več glavnih stavb ter stebrišče ob pristanišču.

Na podlagi rezultatov izkopavanj leta 2008 je mogoče gradnjo severne stavbe s čudovito opremljenimi prostori, tj. glavno stavbo vile, datirati v čas ok. 25-10 pr. Kr. Gradnja se je začela šele po obsežnih izravnavah terena. Na jugu polotoka je še ena stavba z dvoriščem in stebriščem, zgrajena na prehodu v pristanišče s pogledom na morje.

Lega južne strukture glede na severno odstopa za $5^{\circ}$ bolj zahodno. Obe strukturi sta bili zgrajeni v relativnem kronološkem zaporedju, konstrukcija stebrišča na jugu v bližini pristanišča pa je bila zgrajena približno eno generacijo pozneje kot je bila zgrajena stavba na severu.

Glede na rezultate izkopavanj v letih 2008 in 2010 je bila vila zapuščena ok. sredine ali v tretji četrtini I. stoletja (50-70 po Kr.). Zapuščanje bi lahko povzročili endogeni vplivi, tj. potresi ali nevihte.

Po opustitvi struktur so bili sistematično odstranjeni gradbeni materiali (mozaična tla, stene, strešne ploščice); ponovno uporabo gradbenega materiala je mogoče časovno umestit takoj po opustitvi. Sistematični ropanje kamna hipotetično kaže na to, da so se obnove dogajale v bližini pristanišča, ki je bilo še vedno v uporabi.

\section{Abbreviations}

Cassani $=$ Cassani, 1991

Conspectus = Ettlinger et al., 1990

Lamboglia = Lamboglia, 1952
$\mathrm{O}=($ Befund)objekt

ÖAI = Österreichisches Archäologisches Institut

Olcese $=$ Olcese, 2003

ZRS = Znanstveno-raziskovalno središče

\section{Bibliography}

Antonioli, Fabrizio, Marco Anzidei, Kurt Lambeck, Rita Auriemma, Dario Gaddi, Stefano Furlani, Paola Orrù, Emanuela Solinas, Andrej Gaspari, Snježana Karinja, Vladimir Kovačić, and Luciano Surace. »Sea-level Change during the Holocene in Sardinia and in the Northeastern Adriatic (Central Mediterranean Sea) from Archaeological and Geomorphological Data.« Quaternary Science Reviews 26 (2007): 2463-486.

Begović Dvoržak, Vlasta, and Ivančica Dvoržak Schrunk. »Roman Villas in Istria and Dalmatia, Part III: Maritime Villas.« Prilozi Instituta za arheologiju u Zagrebu 2I (2004): 65-90.

Boltin-Tome, Elica. »Arheološke najdbe na kopnem in na morskem dnu v Viližan in Simonovem zalivu v Izoli.« Annales I (199I): $5 \mathrm{I}-58$.

Cassani, Giovanna. »La ceramica della US ı 100 della villa di Pavia di Udine: Relazione preliminare sulla rozza terracotta.« Quaderni Friulani di Archeologia I (I99I): 89-102.

De Franceschini, Marina. Le ville romane della Regio X (Venetia et Histria). Roma: »L'Erma $\ll$ di Bretschneider, 1998.

Degrassi, Attilio. »I porti romani dell'Istria.« In Scritti di Archeologia e di Antichità Classiche in onore di Carlo Anti, 119-69. Firenze: Sansoni, I955.

Degrassi, Attilio. $\gg$ Tracce di Roma sulla spiaggia di San Simone.« Archeografo Triestino Io, no. 3 (1923): 329-4I.

Donderer, Michael. Die Chronologie der römischen Mosaiken in Venetien und Istrien bis zur Zeit der Antonine. Berlin: Mann, 1986. 
Ettlinger, Elisabeth, Bettina Hedinger, Bettina Hoffmann, Philip Kenrick, Giuseppe Pucci, Katrin Roth-Rubi, Gerwulf Schneider, Siegmar von Schnurbein, Colin Wells, and Susanne Zabehlicky-Scheffenegger. Conspectus Formarum Terrae Sigillatae Italico Modo Confectae. Bonn: Habelt, 1990.

Fontana, Federica. La villa romana di Barcola: A proposito delle Villae Maritimae della Regio X. Roma: Quasar, 1993.

Groh, Stephan, and Helga Sedlmayer. »Die Grabungen in der römischen Villa maritima von San Simone/Simonov zaliv bei Isola/Izola, Slowenien.«Annales, Series historia et sociologia 18 no. 2 (2008): 385-96.

Groh, Stephan, and Helga Sedlmayer. »Nuove ricerche nella villa maritima romana di San Simone/Simonov zaliv presso Isola/Izola (Slovenia).« Aquileia Nostra 80 (2010): II3-25.

Karinja, Snježana. »Dve rimski pristanišči v Izoli.« In Arheološka istraživanja u Istri, edited by Božidar Čečuk, I77-92. Zagreb: Hrvatsko arheološko društvo, 1997.

Labud, Giordano. »Studio tipilogico di reperti anforici rinvenuti nel sito archeologico romano di San Simone (Simonov zaliv), nell'Istria settentrionale.« Opuscula romana 20 (1996): 254-60.

Lafon, Xavier. Villa Maritima. Roma: École Française de Rome, 200I.

Lamboglia, Nino. »Per una classificazione preliminare della ceramica campana.« In Atti del primo Congresso internazionale di Studi Liguri 1950, 139-206. Bordighera: Stabilimento Tipigrafico Cune, 1952.

Mikl Curk, Iva. »Simonov zaliv - Terra Sigillata from the first field campaigns. «Opuscula romana 20 (1996): 249-53.

Mušič, Branko. Poročilo o geofizikalni raziskavi: Izola - Simonov zaliv. Ljubljana: Inedito rapporto di prospezione, 2006.

Olcese, Gloria. Ceramiche comuni a Roma e in area romana: produzione, circolazione e tecnologia. Mantova: Società Archeologica Padana, 2003.
Stokin, Marko. »Simonov zaliv: Antična vila.« Annales, Series historia et sociologia II, no. I (200I): 405-I2.

Šribar, Vinko. »Constatations archéologico-topographiques dans la baie de Simon près d'Izola.« Arbeološki Vestnik 9-Io (19581959): 27I-77.

Šribar, Vinko. »Izola.« Varstvo Spomenikov I2 (1969): 89-91. 